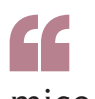

mice

expressing

high levels

of $T L R 8$

spontaneously

developed

arthritis

\title{
Making mice more human the TLR8 way
}

In addition to responding to pathogen-derived ligands, Toll-like receptors (TLRs) can respond to endogenous ligands and thus have been linked with the pathogenesis of autoimmunity. Guiducci et al. have now generated transgenic mice that express human $T L R 8$, and they reveal a role for this receptor in autoimmune disease.

In humans, both TLR7 and TLR8 recognize viral RNA, single-stranded self-RNA and synthetic small molecule agonists. TLR7 has a role

in autoimmune diseases, but although TLR8 belongs to the same TLR family as TLR7, its role in inflammation and disease has been unclear owing to the lack of a suitable animal model.
In mice, TLR8 is non-functional because it lacks five amino acids, so the authors generated transgenic C57BL/6 mice that express different levels of human TLR8. Peripheral blood mononuclear cells isolated from TLR 8 transgenic mice, but not from wild-type mice, were responsive to an RNA-based ligand of human TLR8 and produced pro-inflammatory cytokines such as interleukin-6 (IL-6), IL-1, interferon- $\gamma$ and tumour necrosis factor; hence, human TLR8 is functional when expressed in mice. Mice expressing high levels of TLR8 mRNA developed a wasting disease, showed severe inflammation in the pancreas, salivary glands and joints and failed to breed, whereas mice expressing lower levels of TLR8 bred normally. The severity of the inflammatory phenotypes closely correlated with the expression levels of TLR8.

Previous work has shown that increased expression of mouse TLR7 is sufficient to break immune tolerance in mice. Interestingly, the authors found that high levels of human TLR8 result in a range of diseases that are strikingly distinct from those related to TLR7 signalling. The different responses are probably due to the fact that TLR7 is primarily expressed by $\mathrm{B}$ cells and plasmacytoid dendritic cells, whereas TLR 8 is expressed by monocytes, myeloid dendritic cells and neutrophils.

Next, the authors examined the development of arthritis in TLR8 transgenic mice; mice expressing high levels of TLR8 spontaneously developed arthritis, whereas mice expressing low levels of TLR $8 \mathrm{did}$ not. However, in contrast to wildtype mice, the disease progression of collagen-induced arthritis continued beyond 4 weeks in mice expressing low levels of TLR8. Hence, TLR8 signalling seems to have a role in the progression and exacerbation of collagen-induced arthritis rather than in the initial phase of the disease.

Finally, the authors determined the level of TLR 8 mRNA in blood cells from patients with arthritis and found that patients with systemic-onset juvenile arthritis or Still's disease had increased levels of TLR8 expression. TLR8 levels in these patients correlate with increased expression of IL- $1 \beta$, which is an important driver of arthritis. Together, these data suggest that human TLR8 has an important role in the pathogenesis of arthritis.

Elisabeth Kugelberg

ORIGINAL RESEARCH PAPER Guiducci, C. et al.

RNA recognition by human TLR 8 can lead to autoimmune inflammation. J. Exp. Med. http://dx.doi.org/10.1084/jem.20131044 (2013) 